\title{
Highly Sensitive Acetylene Sensing Properties of Al- and In-doped ZnO Quantum Dots
}

\author{
Min Sun Park, Ran Yoo, Byungjin Jang, Yunji Park, Min Hyung Kim, Hyun-Sook Lee, Wooyoung Lee† \\ Department of Materials Science and Engineering, Yonsei University, \\ 50 Yonsei-ro, Seodaemun-gu, Seoul, 03722, Republic of Korea \\ ${ }^{*}$ E-mail: wooyoung@yonsei.ac.kr (W. Lee)
}

\begin{abstract}
Acetylene $\left(\mathrm{C}_{2} \mathrm{H}_{2}\right)$, dissolved in oil-filled power equipment, has undoubtedly the most detrimental risk in terms of safety owing to the arc discharge characteristics. It is necessary to systematically detect the dissolved acetylene gas for optimum operation condition which can directly affect the safety and stability of the power system. In this vein, we report the high-performance $\mathrm{C}_{2} \mathrm{H}_{2}$ gas sensor based on 1 at\% In-doped $\mathrm{ZnO}$ quantum dots (QDs) which was synthesized by a hydrothermal method. The phase and morphology of the as-synthesized QDs were characterized by X-ray diffraction (XRD) and transmission electron microscope (TEM) analyses. The sensing properties of the $\mathrm{C}_{2} \mathrm{H}_{2}$ gas were carried out by exposing the sensor to various concentration under the various working temperatures. The response to the $10 \mathrm{ppm}$ acetylene was $\sim 314$ in air and $\sim 570$ in $\mathrm{N}_{2}$ at the optimum operating temperature, which are superior to that of other previously reported $\mathrm{C}_{2} \mathrm{H}_{2}$ sensors based on semiconducting metal oxides.
\end{abstract}

Key words: acetylene gas sensor, In-doped $\mathrm{ZnO}$, quantum dots, transformer oil

\section{Introduction}

One of the most challenging ongoing issues in power transformers is the monitoring of degradation in the internal components of a transformer. When the oil-filled-transformer is under abnormal stress, it can lead to the chemical breakdown of the oil or cellulose, disenabling the dielectric insulation. The failure to transfer leads to the evolution of a mixture of gases, including hydrogen $\left(\mathrm{H}_{2}\right)$, hydrocarbons $\left(\mathrm{CH}_{4}, \mathrm{C}_{2} \mathrm{H}_{2}, \mathrm{C}_{2} \mathrm{H}_{4}, \mathrm{C}_{2} \mathrm{H}_{6}\right)$, and carbon oxides $\left(\mathrm{CO}, \mathrm{CO}_{2}\right)$. Detection of the generated gases can be one of the markers to diagnose the failure or predict the lifespan of a transformer. Among the gases that can be generated in transformer oil, $\mathrm{C}_{2} \mathrm{H}_{2}$ is the most important gas to be monitored in a transformer due to its characteristic to evolve during arc discharge, which leads to the catastrophic accidents of a transformer [1]. In this work, we report the highly sensitive $\mathrm{C}_{2} \mathrm{H}_{2}$ sensing performance of Al- (AZO) and In-doped (IZO) ZnO QDs. The comparison of sensing performance with previously reported results in other groups and the sensing mechanism are addressed.
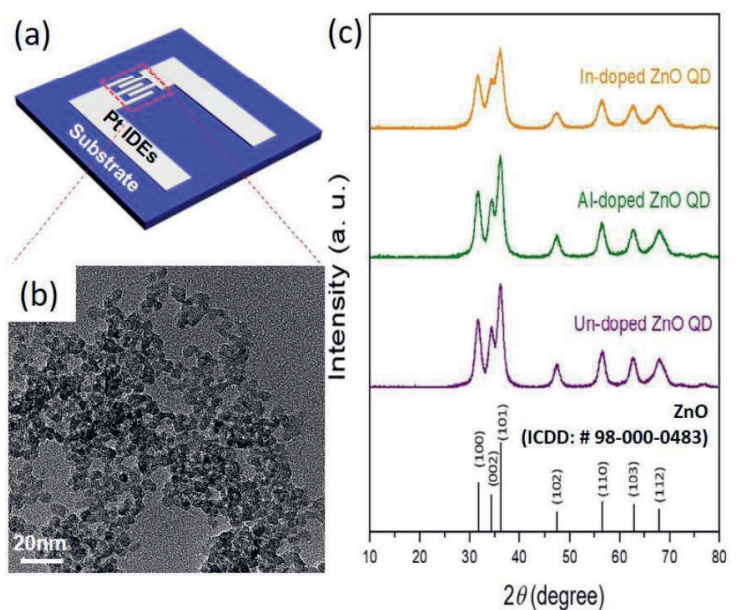

Fig. 1. (a) Schematic illustration of the sensor device; (b) TEM image of actual 1 at\% In-doped ZnO QDs; (c) XRD patterns of un-doped, Al-, and In-doped $\mathrm{ZnO}$ QDs.

\section{Results and Discussion}

Un-doped $\mathrm{ZnO}(\mathrm{ZO})$ and doped (Al, In) $\mathrm{ZnO}$ QDs were synthesized by a wet chemical method as described in our previous reports [2]. Figure 1(a) shows a schematic image of an actual sensor device composed of Pt electrodes interdigitated on a $\mathrm{SiO}_{2}$ substrate via a photolithography method. Figure 1(b) presents the TEM image of the as-synthesized IZO QDs. 
In the magnified position of the device in Fig. $1(\mathrm{a})$, a ZnO QD layer is deposited on top of the $\mathrm{Pt}$ electrodes. The XRD patterns confirm that the nanocrystals are of crystallinity with the typical hexagonal wurtzite crystal structure $\mathrm{ZnO}$ (see Fig. 1(c)). The broadening of the peaks in the XRD patterns can be attributed to the small particle size $(\sim 5 \mathrm{~nm})$ of the as-synthesized $\mathrm{ZnO}$ QDs. The real-time electrical resistance of $Z O$, $\mathrm{AZO}$, and IZO QDs were measured at various $\mathrm{C}_{2} \mathrm{H}_{2}$ concentrations in air and $\mathrm{N}_{2}$, respectively. The sensing response of the $\mathrm{C}_{2} \mathrm{H}_{2}$ gas is defined as $\left(R_{\mathrm{a}}-R_{\mathrm{g}}\right) / R_{\mathrm{g}}$, where $R_{\mathrm{a}}$ and $R_{\mathrm{g}}$ are the resistance of the sensors in air $\left(\mathrm{N}_{2}\right)$ and in the environment containing acetone, respectively.

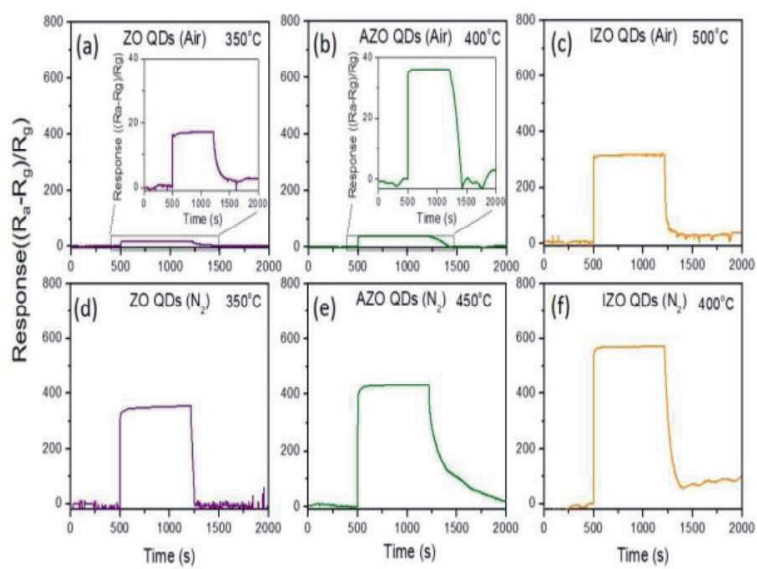

Fig. 2. Variation in sensing responses of $Z O, A Z O$, IZO QDs at $10 \mathrm{ppm} \quad \mathrm{C}_{2} \mathrm{H}_{2}$ under their optimal operating temperatures in (a)-(c) air and (d)-(f) $N_{2}$, respectively.

Figure 2 shows the variation in responses of $Z O$, $\mathrm{AZO}$, and IZO QDs to 10ppm $\mathrm{C}_{2} \mathrm{H}_{2}$ under optimized working temperature in air and $\mathrm{N}_{2}$, respectively. In both air and $\mathrm{N}_{2}$, a higher sensing performance is observed with $A Z O$ QDs compared to ZO QDs and with IZO QDs compared to $A Z O Q D$. This is due to the replacement of the $\mathrm{Zn}^{2+}$ cation by $\mathrm{Al}^{3+}$ and $\mathrm{In}^{3+}$, which act as a donor, leads to formation of active adsorption sites which favor the adsorption of oxygen species.

The best sensing response is observed in IZO QDs. The maximum responses of IZO QDs are $\sim 314$ in air and $\sim 570$ in $N_{2}$, which are superior to that of other previously reported $\mathrm{C}_{2} \mathrm{H}_{2}$ sensor based on semiconducting metal oxides [3-6]. This can be attributed to a greater number of absorbed oxygen ion species, providing more active adsorption sites, in IZO QDs compared to $\mathrm{ZO}$ and $\mathrm{AZO} \mathrm{QDs}$. The response as a function of $\mathrm{C}_{2} \mathrm{H}_{2}$ concentrations and analyses from X-ray photoelectron spectroscopy (XPS), UV-visible spectrophotometry (UV-vis), and Brunauer-Emmett-Teller (BET) are presented in detail.
Table. 1. Sensing properties of various metal oxide semiconductor gas sensors to $\mathrm{C}_{2} \mathrm{H}_{2}(\mathrm{~S}=$ sensitivity (response/ppm); L=low detection limit; $t_{R}=$ response time; $T_{\text {opt }}=$ optimal working temperature)

\begin{tabular}{|l|c|c|c|c|c|}
\hline & {$[A]$} & {$[B]$} & {$[C]$} & {$[D]$} & {$[E]$} \\
\hline$S$ & 7.9 & 1.0 & 0.14 & 0.12 & 31.4 \\
\hline$L(p p m)$ & 1 & 1 & 1 & 3 & 0.1 \\
\hline$t_{R}(s)$ & 15 & 5 & 2 & 57 & 5 \\
\hline$T_{\text {opt }}\left({ }^{\circ} \mathrm{C}\right)$ & 420 & 250 & 206 & 200 & 500 \\
\hline
\end{tabular}

[A] An ZnO microdisks [3]

[B] Ni-doped ZnO nanoflowers [4]

[C] $\mathrm{NiO} / \mathrm{SnO}_{2}$ heterostructures [5]

[D] Ag/ZnO Hrc-RGO hybrid [6]

[E] IZO QDs (this work)

\section{References}

[1] Byungjin Jang, Min Hyung Kim, Jisun Baek, Wonkung Kim, Wooyoung Lee. Highly Sensitive Hydrogen Sensors: Pd-coated Si Nanowire Arrays for Detection of Dissolved Hydrogen in Oil. Sens. Actuators B 256 (2018) 465-471 DOI: 10.1016/j.snb.2017.10.109

[2] R. Yoo, D. Lee, S. Cho, W. Lee. Doping effect on the sensing properties of $\mathrm{ZnO}$ nanoparticles for detection of 2-chloroethyl ethylsulfide as a mustard simulant, Sens. Actuators B: Chem. 254, 1242-1248(2018);DOI:10.1016/j.snb.2017.07.084

[3] Lexi Zhanga,b, Jianghong Zhaoa, Jianfeng Zhenga, Li Li a, Zhenping Zhu. Hydrothermal synthesis of hierarchical nanoparticle-decorated $\mathrm{ZnO}$ microdisks and the structure-enhanced acetylene sensing properties at high temperatures, Sens. Actuators B 158 (2011) 144150 DOI: 10.1016/j.snb.2011.05.057

[4] Xin chang Wanga,n, Minggang Zhaoa, Fang Liua, Jianfeng Jiaa, XinjianLia, Liangliang Cao. C2H2 gas sensor based on Ni-doped ZnO electrospun nanofibers, Ceramic International 39 (2013) 2883-2887, DOI: 10.1016/j.ceramint.2012.09.062

[5] Ying Lin, Chao Li, Wei Wei, Yujia Li, Shanpeng Wen, Dongming Sun, Yu Chen and Shengping Ruan, A new type of acetylene gas sensor based on a hollow heterostructure, RSC Adv., 2015, 5, 61521, DOI: 10.1039/C5RA10327D

[6] A.S.M.Iftekhar Uddin, Kwan-WooLee, Gwiy-Sang Chung, Acetylene gas sensing properties of an Ag-loaded hierarchical $\mathrm{ZnO}$ nanostructuredecorated reduced graphene oxide hybrid, Sens. Actuators B 216 (2015) 33-40, DOI: 10.1016/j.snb.2015.04.028 